Article

\title{
Laboratory Determined Sugar Content and Composition of Commercial Infant Formulas, Baby Foods and Common Grocery Items Targeted to Children
}

\author{
Ryan W. Walker ${ }^{1}$ and Michael I. Goran ${ }^{2, *}$ \\ ${ }^{1}$ Department of Preventive Medicine, Icahn School of Medicine at Mount Sinai, New York, NY 10025, \\ USA; E-Mail: ryan.walker@mssm.edu \\ ${ }^{2}$ Department of Preventive Medicine, University of Southern California, Keck School of Medicine, \\ Los Angeles, CA 90089, USA \\ * Author to whom correspondence should be addressed; E-Mail: goran@usc.edu; \\ Tel.: +1-323-442-3027; Fax: +1-323-442-4103.
}

Received: 28 March 2015 / Accepted: 6 July 2015 / Published: 16 July 2015

\begin{abstract}
Excess added sugar consumption is tied to poor health outcomes in children. The sugar content of beverages and foods children are exposed to is mostly unknown, yet this information is imperative for understanding potential risks from overconsumption of sugars in early life. We determined actual sugar content by conducting a blinded laboratory analysis in infant formulas, breakfast cereals, packaged baked goods and yogurts. One hundred samples were sent to an independent laboratory for analysis via gas chromatography. Sugar content and composition was determined and total sugar was compared against nutrition labels. Of the 100 samples analyzed, $74 \%$ contained $\geqslant 20 \%$ of total calories per serving from added sugars. Nutrient label data underestimated or overestimated actual sugars and $\sim 25 \%$ of all samples had actual total sugar values that were either $<10 \%$ or $>10 \%$ of labeled total sugar. Many products that are frequently marketed to and consumed by infants and young children contain sugars in amounts that differ from nutrition labels and often in excess of recommended daily levels. These findings provide further support for adding more comprehensive sugar labeling to food and beverage products, specifically those marketed to, or commonly consumed by, children.
\end{abstract}

Keywords: sugar; formula; high fructose corn syrup; HFCS; obesity; fructose; breastfeeding 


\section{Introduction}

Nearly $15 \%$ of the United States population consumes upwards of $25 \%$ of their total daily calories from added sugars [1]. Excess consumption of sugar sweetened beverages, a major source of added sugars, is tied to poor health outcomes in children [2], but intake is commonly measured via diet recalls or food frequency questionnaires that assume sugar values based on databases created from product nutrition labels [3-5]. There is very limited data from laboratory-determined measures of sugar content and little is known about the actual sugar content of common processed foods that children may be exposed to very early in life.

Nutrition and dietary habits during infancy and into early childhood play a role in shaping eating habits and health later in adolescence and into adulthood [6,7]. The effect of breastfeeding versus formula feeding on child health outcomes has been studied extensively and is it well established that human milk and infant formulas differ in terms of both nutrition and biological constituents [8,9]. Some formulas contain added sugars that are not present in breastmilk and the actual sugar content, in terms of both type and proportion, of infant formula is not widely known.

As children are introduced to solid foods at weaning, they may be exposed to additional processed food products that contain added sugars [10]. Like some formulas, solid foods may contain sucrose and other sugars that are not present in breastmilk. Commercial baby foods and other common grocery items that children are often exposed to in infancy can be a source of added sugars, which contribute to total daily sugar exposure. Nutrition labels for some commercial products may not always reflect the true, or most accurate, sugar content information [11,12]. Given the recent scientific, federal and consumer interest in the sugar content of foods and beverages, specifically added sugars, it is important to establish actual sugar content and composition for infant formulas and other food products children may be exposed to in early life.

Therefore, we sought to determine actual sugar content and composition, by conducting a blinded gas chromatography analysis, in 20 commonly used infant formulas, 20 baby foods and 60 other common grocery items. Several products frequently marketed towards children, based upon advertising and product packaging [13], were included in the analyses. The additional grocery categories were breakfast cereals, pre-packaged baked goods and yogurts.

\section{Materials and Methods}

One hundred food and beverage samples were selected from infant formulas and other standard grocery categories: Baby food, yogurt, breakfast cereal, and packaged baked goods. Online shopping databases for three of the Nation's largest grocery retailers-Walmart, SuperValu, and Safeway-were accessed in order to select category-specific samples. To control for location and inventory, online store inventories were accessed for selected Los Angeles County outlets of each retailer in a defined zip code region (90033). Twenty products were selected for each of the grocery categories by choosing every tenth product in the retailers' databases until 10 products made with high fructose corn syrup (HFCS) and 10 products made without HFCS, according to package ingredient labels, were selected. In categories where HFCS was not a commonly occurring ingredient, 10 sucrose-containing products and 10 non-sucrose-containing products, according to package ingredients labels, were selected using 
the same method whenever possible. An aliquot was taken directly from each product, in its original packaging, and transferred to sterile, sealed containers. Sample weights were determined and recorded. Sample weights ranged from 15 to $40 \mathrm{~g}$. Samples were packaged and shipped overnight on dry ice to Covance Laboratories (Madison, WI, USA) for subsequent blinded analysis via gas chromatography (Agilent $6890 \mathrm{~N}$ ), against internal standards, according to previously published methods [14-16].

The sugar profile analysis conducted at Covance was applicable to the determination of fructose, galactose, glucose, sucrose, lactose, and maltose in as little as $10 \mathrm{~g}$ of food products, syrups, and beverages. Once received, samples were prepared in accordance with Covance procedures and sugars were extracted from the homogenized sample with water. Aliquots were dried under inert gas and reconstituted with a hydroxylamine hydrochloride solution in pyridine containing phenyl- $\beta$-D-glucoside as the internal standard. The resulting oximes were converted to silyl derivatives with hexamethyldisilazane and trifluoracetic acid treatment and analyzed by gas chromatography $[15,16]$ using a flame ionization detector. This methodology does not acid hydrolyze or purposefully degrade sugars during analysis, thereby mitigating the risk of more complex sugars degrading during analysis (the sugars are in solution and the extracting solvents inhibit enzymatic activity). All GC sample analyses were conducted with an internal standard (Phenyl-Beta-D-Glucopyranoside). An additional 10\% of each sample analytical run was tested in duplicate and validated against two internally-validated control standards. The limit of quantitation for most matrices is $0.1 \%$. A cereal product was included in daily batch analyses as a routine control material. A control chart was created for these results and values were used as a quality control parameter for the acceptance of GC assay data. RSDs (relative standard deviation) were assembled from many data points and provide a reliable representation of the variance for the cereal product matrix across days, instruments and analysts. The cereal product RSDs for fructose, glucose, sucrose, and maltose were 4.9, 7.4, 3.2, and 6.4, respectively. Results underwent further quality control comparison with internal validated controls, linearity expectations and historical data.

\section{Analysis}

Sugar data was provided in a value of grams per $100 \mathrm{~g}$ of sample, as per industry standard [17]. Data for individual sugars were converted for reporting into the following formats; percent of total sugar and concentration of each sugar in grams per serving $(\mathrm{g} / \mathrm{s})$. Actual total sugar was calculated. The percent of total calories per serving (based upon nutrition label values, where available) from sugar was calculated. Formulas used to obtain these values are presented in Table S1. Products with "added sugar" listed one or more of the following sugars in the product nutrition label ingredients; HFCS, sucrose, fructose, glucose, lactose, glucose, galactose, maltose or corn syrup. The percent of total sugar from "added" $v s$. "intrinsic" sources was determined (Table S2). The percent of total sugar from added sugar is equal to the percentage sum of all measured sugars that were listed in the product ingredients. The percent of total sugar from intrinsic sugar is equal to all other measured sugars (not listed in product ingredients). In products listing "sugar" as an ingredient, sucrose was included as an added sugar. Maltose was included as an added sugar in items listing corn syrup as an ingredient. Glucose and fructose were included as added sugars in products listing HFCS on the label. In fruit-based products containing HFCS it was not possible to determine proportions of fructose and glucose from added $v s$. intrinsic sources. 


\section{Results}

\subsection{Sugar Analyses Using Gas Chromatography in 100 Foods and Beverages}

\subsubsection{Infant Formula}

Mean percent of total calories per serving from sugars was $27.4 \% \pm 13.6 \%$. Five formulas had $>40 \%$ of total calories from sugars (Figure 1). Sugar content was predominantly distributed amongst lactose, sucrose, maltose or glucose $(77.5 \pm 34,68.6 \pm 28.5,19.1 \pm 24.7$ and $6.6 \pm 10.1 \%$, respectively), in products that contained said sugars, with little to no free fructose or galactose detected in most products (Table 1). Formulas highest in sucrose were alternatives to lactose-containing milk-based formulas. All but two formulas listed sugars on the ingredients label, thus qualifying them as products with added sugar. Among products with added sugar mean percent of total sugar from added sugar sources was $69.9 \pm 37.4 \%$ (Table S2). Grams of total sugars per serving ranged from 1.28 to $11.16 \mathrm{~g}$, with five products containing $>10 \mathrm{~g}$ of sugar per serving (Table S3).

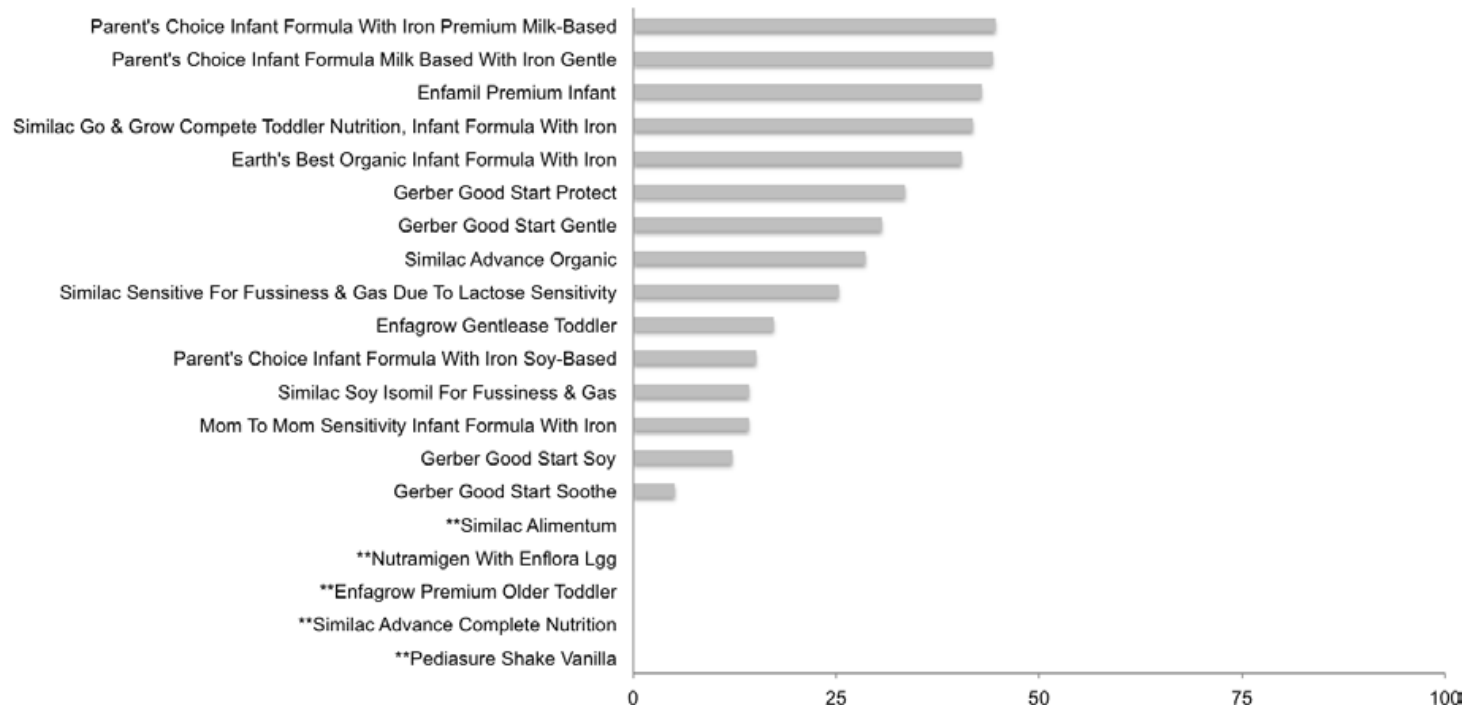

Figure 1. Percent of total calories from sugar: Formula. ** Unable to calculate in premixed, ready to use samples. Values were derived from the following equation: \% Total Calories from sugar $=\left[\left(\right.\right.$ Total Sugar $_{\text {Actual }}$ per serving $\left.\times 4\right) /$ Total Calories per serving $] \times 100$.

\subsubsection{Baby Food}

Mean percent of total calories per serving from sugars was $38.6 \pm 25.5 \%$ and seven of the 20 baby food products contained $>50 \%$ of their total calories from sugar (Figure 2) with First Foods Gerber Select Apples having $87.6 \%$ of its total calories from sugar. Several of the fruit-based products had $>40 \%$ of total sugars from free fructose (Table 1), with First Foods Gerber Select Apples having 68.5\% of total sugars from free fructose. The remainder of baby foods tested had free fructose levels below 55\%. Seven baby foods listed sugars on the ingredients label and the mean percent of total sugar from added sugar sources was $53.6 \pm 24.1 \%$ (Table S2). Gerber Spoonable Smoothies Hawaiian Delight contained $20.7 \mathrm{~g}$ of total sugars per serving and six other products has $>10 \mathrm{~g} / \mathrm{s}$ of sugar (Table S3). Only one product listed HFCS as a sweetener. 
Table 1. Percentage of Total Sugar.

\begin{tabular}{|c|c|c|c|c|c|c|}
\hline Formula & $\begin{array}{c}\% \\
\text { Fructose }\end{array}$ & $\begin{array}{c}\% \\
\text { Glucose }\end{array}$ & $\begin{array}{c}\% \\
\text { Sucrose }\end{array}$ & $\begin{array}{c}\% \\
\text { Maltose }\end{array}$ & $\begin{array}{c}\% \\
\text { Galactose }\end{array}$ & $\begin{array}{c}\% \\
\text { Lactose }\end{array}$ \\
\hline Pediasure Shake Vanilla & 0.00 & 1.37 & 98.63 & 0.00 & 0.00 & 0.00 \\
\hline Similac Alimentum & 0.00 & 0.00 & 100.00 & 0.00 & 0.00 & 0.00 \\
\hline Enfagrow Premium Older Toddler & 0.00 & 1.85 & 14.81 & 0.00 & 0.00 & 83.33 \\
\hline Similac Advance Complete Nutrition & 0.00 & 1.59 & 0.00 & 0.00 & 0.00 & 98.41 \\
\hline $\begin{array}{l}\text { Similac Go \& Grow Compete Toddler } \\
\text { Nutrition, Infant Formula With Iron }\end{array}$ & 0.00 & 2.08 & 0.00 & 0.00 & 0.21 & 97.71 \\
\hline $\begin{array}{l}\text { Earth's Best Organic Infant Formula With } \\
\text { Iron }\end{array}$ & 0.00 & 0.65 & 0.00 & 3.44 & 0.00 & 95.91 \\
\hline Nutramigen With Enflora Lgg & 0.00 & 42.22 & 0.00 & 57.78 & 0.00 & 0.00 \\
\hline Enfagrow Gentlease Toddler & 0.00 & 10.05 & 0.00 & 11.06 & 0.50 & 78.39 \\
\hline Gerber Good Start Soothe & 0.00 & 20.34 & 0.00 & 79.66 & 0.00 & 0.00 \\
\hline $\begin{array}{l}\text { Parent's Choice Infant Formula with Iron } \\
\text { Soy-Based }\end{array}$ & 0.00 & 1.72 & 0.00 & 4.60 & 2.87 & 90.80 \\
\hline $\begin{array}{c}\text { Parent's Choice Infant Formula with Iron } \\
\text { Premium Milk-Based }\end{array}$ & 0.00 & 1.75 & 0.00 & 0.00 & 0.19 & 98.05 \\
\hline $\begin{array}{c}\text { Parent's Choice Infant Formula Milk } \\
\text { Based with Iron Gentle }\end{array}$ & 0.00 & 1.77 & 0.00 & 0.00 & 0.20 & 98.04 \\
\hline Gerber Good Start Protect & 0.00 & 1.04 & 0.00 & 1.30 & 1.04 & 96.62 \\
\hline Gerber Good Start Gentle & 0.00 & 3.69 & 0.00 & 1.70 & 1.70 & 92.90 \\
\hline Enfamil Premium Infant & 0.00 & 1.22 & 0.00 & 0.00 & 0.41 & 98.38 \\
\hline $\begin{array}{c}\text { Mom To Mom Sensitivity Infant Formula } \\
\text { With Iron }\end{array}$ & 0.56 & 6.67 & 63.89 & 24.44 & 0.00 & 4.44 \\
\hline Similac Advance Organic & 0.61 & 1.52 & 44.68 & 3.34 & 0.00 & 49.85 \\
\hline $\begin{array}{l}\text { Similaac Sensitive For Fussiness \& Gas } \\
\text { Due To Lactose Sensitivity }\end{array}$ & 0.69 & 9.28 & 79.73 & 8.25 & 0.00 & 2.06 \\
\hline Gerber Good Start Soy & 0.71 & 3.57 & 82.14 & 13.57 & 0.00 & 0.00 \\
\hline Similac Soy Isomil For Fussiness \& Gas & 1.22 & 14.02 & 64.63 & 20.12 & 0.00 & 0.00 \\
\hline Yogurt & $\begin{array}{c}\% \\
\text { Fructose }\end{array}$ & $\begin{array}{c}\% \\
\text { Glucose }\end{array}$ & $\begin{array}{c}\% \\
\text { Sucrose }\end{array}$ & $\begin{array}{c}\% \\
\text { Maltose }\end{array}$ & $\begin{array}{c}\% \\
\text { Galactose }\end{array}$ & $\begin{array}{c}\% \\
\text { Lactose }\end{array}$ \\
\hline Dannon Oikos Blueberry & 46.09 & 9.38 & 24.22 & 0.00 & 4.69 & 15.63 \\
\hline Great Value N.F. Strawberry Banana & 42.37 & 3.39 & 0.00 & 0.00 & 11.86 & 42.37 \\
\hline Dannon Oikos Greek N.F. Vanilla & 40.17 & 2.56 & 36.75 & 0.00 & 5.13 & 15.38 \\
\hline Activia Light Strawberry & 30.99 & 4.23 & 2.82 & 0.00 & 5.63 & 56.34 \\
\hline Activia Vanilla & 27.97 & 2.80 & 44.76 & 0.00 & 2.80 & 21.68 \\
\hline Lucerne L.F. Tubes Blueberry Bubblegum & 26.28 & 1.28 & 44.87 & 0.00 & 2.56 & 25.00 \\
\hline Lucerne L.F. Tubes Strawberry & 25.81 & 0.65 & 45.81 & 0.00 & 2.58 & 25.16 \\
\hline * Yoplait Whips! Chocolate & 20.71 & 9.09 & 48.48 & 0.00 & 2.53 & 19.19 \\
\hline Lucerne L.F. Yogurt Peach & 11.03 & 9.56 & 57.35 & 0.00 & 3.68 & 18.38 \\
\hline Chobani Raspberry & 10.26 & 8.55 & 58.97 & 0.00 & 4.27 & 17.95 \\
\hline Yoplait Light Blackberry & 3.70 & 3.70 & 37.04 & 0.00 & 7.41 & 48.15 \\
\hline Yoplait Original Strawberry & 2.50 & 2.50 & 72.50 & 0.00 & 3.13 & 19.38 \\
\hline $\begin{array}{c}\text { Dannon Danimal strawberry-Banana } \\
\text { Flavored }\end{array}$ & 2.19 & 2.19 & 76.64 & 0.00 & 3.65 & 15.33 \\
\hline Yoplait Gogurt Perry Berry & 1.17 & 1.17 & 79.53 & 0.00 & 2.92 & 15.20 \\
\hline
\end{tabular}


Table 1. Cont.

\begin{tabular}{|c|c|c|c|c|c|c|}
\hline Yoplait Trix Strawberry Banana Bash & 0.74 & 1.47 & 72.79 & 0.00 & 2.94 & 22.06 \\
\hline Yoplait Gogurt Summer Punch & 0.58 & 1.16 & 79.77 & 0.00 & 2.89 & 15.61 \\
\hline Yoplait Light Key Lime Pie & 0.00 & 0.00 & 39.29 & 0.00 & 7.14 & 53.57 \\
\hline Yoplait Light Banana Cream Pie & 0.00 & 1.54 & 40.00 & 0.00 & 7.69 & 50.77 \\
\hline Yoplait Light Very Vanilla & 0.00 & 1.59 & 39.68 & 0.00 & 7.94 & 50.79 \\
\hline Baked Goods & $\begin{array}{c}\% \\
\text { Fructose }\end{array}$ & $\begin{array}{c}\% \\
\text { Glucose }\end{array}$ & $\begin{array}{c}\% \\
\text { Sucrose }\end{array}$ & $\begin{array}{c}\% \\
\text { Maltose }\end{array}$ & $\begin{array}{c}\% \\
\text { Galactose }\end{array}$ & $\begin{array}{c}\% \\
\text { Lactose }\end{array}$ \\
\hline * Keebler 100 Calorie Right Bites & 46.88 & 17.81 & 27.81 & 5.94 & 0.00 & 1.56 \\
\hline Kellogg’s Special K Pastry & 33.57 & 12.94 & 45.80 & 6.64 & 0.35 & 0.70 \\
\hline * Nabisco Fig Newtons & 29.01 & 35.62 & 26.72 & 8.14 & 0.51 & 0.00 \\
\hline Fiber One 90 Calorie Brownies & 22.61 & 1.91 & 75.16 & 0.32 & 0.00 & 0.00 \\
\hline * Kellogg's Pop-Tarts Strawberry & 14.38 & 52.61 & 22.88 & 10.13 & 0.00 & 0.00 \\
\hline$*$ Hostess Twinkies & 11.23 & 33.68 & 42.82 & 9.92 & 0.00 & 2.35 \\
\hline * Little Debbie Muffins Blueberry & 8.38 & 17.30 & 71.62 & 1.08 & 0.00 & 1.62 \\
\hline * Safeway Graham Crackers & 8.24 & 7.84 & 82.75 & 0.00 & 1.18 & 0.00 \\
\hline Entenmann’s Little Bites & 7.09 & 2.75 & 84.67 & 3.20 & 0.00 & 2.29 \\
\hline * Nabisco Nilla Wafers & 4.48 & 5.07 & 86.87 & 0.00 & 0.00 & 3.58 \\
\hline * Bimbo Mini Muffins & 3.65 & 4.38 & 89.78 & 0.36 & 0.00 & 1.82 \\
\hline * Hostess Sno Balls & 3.60 & 18.65 & 65.62 & 11.46 & 0.00 & 0.67 \\
\hline * Little Debbie Cosmic Brownies & 2.65 & 38.73 & 46.95 & 11.67 & 0.00 & 0.00 \\
\hline * Mini Oreo Bite Size & 2.61 & 10.97 & 86.42 & 0.00 & 0.00 & 0.00 \\
\hline * Mini Chips Ahoy Bite Size & 1.93 & 4.18 & 93.89 & 0.00 & 0.00 & 0.00 \\
\hline Hostess Ho Hos & 1.79 & 4.56 & 88.10 & 2.78 & 0.00 & 2.78 \\
\hline Entenmann's Buttermilk Donuts & 0.99 & 2.47 & 94.32 & 0.74 & 0.00 & 1.48 \\
\hline * Hostess Donettes Crunch & 0.92 & 3.99 & 89.88 & 3.99 & 0.00 & 1.23 \\
\hline Hostess Donettes & 0.74 & 41.91 & 52.94 & 2.57 & 0.00 & 1.84 \\
\hline Cereals & $\begin{array}{c}\% \\
\text { Fructose } \\
\end{array}$ & $\begin{array}{c}\% \\
\text { Glucose } \\
\end{array}$ & $\begin{array}{c}\% \\
\text { Sucrose } \\
\end{array}$ & $\begin{array}{c}\% \\
\text { Maltose } \\
\end{array}$ & $\begin{array}{c}\% \\
\text { Galactose } \\
\end{array}$ & $\begin{array}{c}\% \\
\text { Lactose } \\
\end{array}$ \\
\hline Cinnamon Toast Crunch & 31.39 & 5.50 & 63.11 & 0.00 & 0.00 & 0.00 \\
\hline Corn Flakes & 15.48 & 14.29 & 59.52 & 10.71 & 0.00 & 0.00 \\
\hline * Essential Everyday Crispy Rice & 8.24 & 9.41 & 78.82 & 3.53 & 0.00 & 0.00 \\
\hline Honey Nut Cheerios & 6.07 & 6.07 & 87.86 & 0.00 & 0.00 & 0.00 \\
\hline Kellogg's Special K & 4.13 & 3.31 & 86.78 & 4.96 & 0.00 & 0.83 \\
\hline Kix & 3.96 & 2.97 & 93.07 & 0.00 & 0.00 & 0.00 \\
\hline Golden Grahams & 3.70 & 3.99 & 91.74 & 0.00 & 0.57 & 0.00 \\
\hline Honey Bunches Of Oats & 3.68 & 5.26 & 81.58 & 8.42 & 0.00 & 1.05 \\
\hline Kashi Go Lean Crunch & 3.64 & 6.36 & 76.36 & 13.64 & 0.00 & 0.00 \\
\hline Kellogg's Frosted Flakes & 3.27 & 3.78 & 91.94 & 1.01 & 0.00 & 0.00 \\
\hline Safeway Crispy Rice & 2.86 & 3.81 & 89.52 & 3.81 & 0.00 & 0.00 \\
\hline Rice Chex & 2.70 & 2.70 & 94.59 & 0.00 & 0.00 & 0.00 \\
\hline Cocoa Puffs & 2.54 & 6.09 & 87.56 & 3.81 & 0.00 & 0.00 \\
\hline Cheerios & 2.50 & 0.00 & 97.50 & 0.00 & 0.00 & 0.00 \\
\hline Trix & 2.48 & 10.56 & 81.37 & 5.59 & 0.00 & 0.00 \\
\hline Lucky Charms & 2.06 & 13.53 & 79.59 & 4.82 & 0.00 & 0.00 \\
\hline Frosted Cheerios & 1.16 & 4.64 & 91.88 & 2.32 & 0.00 & 0.00 \\
\hline Frosted Mini Wheats Little Bites & 1.00 & 1.50 & 95.00 & 2.50 & 0.00 & 0.00 \\
\hline Kellogg's Froot Loops & 0.98 & 1.22 & 97.80 & 0.00 & 0.00 & 0.00 \\
\hline Life & 0.94 & 1.41 & 93.90 & 3.76 & 0.00 & 0.00 \\
\hline
\end{tabular}


Table 1. Cont.

\begin{tabular}{ccccccc}
\hline Baby Foods & $\begin{array}{c}\% \\
\text { Fructose }\end{array}$ & $\begin{array}{c}\% \\
\text { Glucose }\end{array}$ & $\begin{array}{c}\% \\
\text { Sucrose }\end{array}$ & $\begin{array}{c}\text { \% } \\
\text { Maltose }\end{array}$ & $\begin{array}{c}\% \\
\text { Galactose }\end{array}$ & $\begin{array}{c}\text { \% } \\
\text { Lactose }\end{array}$ \\
\hline Gerber Nature Select Apples & 68.52 & 23.15 & 8.33 & 0.00 & 0.00 & 0.00 \\
Gerber Prunes With Apples & 54.17 & 42.50 & 3.33 & 0.00 & 0.00 & 0.00 \\
Gerber Smoothies Peach Cobbler & 46.72 & 40.16 & 13.11 & 0.00 & 0.00 & 0.00 \\
Gerber Breakfast Apple Cinnamon & 42.17 & 31.33 & 26.51 & 0.00 & 0.00 & 0.00 \\
Gerber Smoothies Hawaiian Delight & 39.05 & 35.24 & 18.10 & 0.00 & 0.48 & 7.14 \\
Gerber Grabbers Fruit \& Yogurt & 34.78 & 18.26 & 34.78 & 0.00 & 2.61 & 9.57 \\
Earth's Best Second Carrots & 24.44 & 24.44 & 51.11 & 0.00 & 0.00 & 0.00 \\
* Gerber Cereal Bars Strawberry Banana & 22.98 & 32.11 & 42.04 & 2.35 & 0.00 & 0.52 \\
O Organics (1) Organic Carrots & 13.51 & 16.22 & 70.27 & 0.00 & 0.00 & 0.00 \\
Beech Nut Tender Sweet Carrots & 13.16 & 13.16 & 73.68 & 0.00 & 0.00 & 0.00 \\
Gerber Juice Treats Fruit Medley & 12.64 & 20.69 & 59.93 & 6.73 & 0.00 & 0.00 \\
Gerber Cereal Twists Banana Peach & 10.65 & 19.35 & 61.94 & 5.48 & 0.00 & 2.58 \\
Gerber Puffs Cereal Snack Peach & 10.12 & 3.57 & 86.31 & 0.00 & 0.00 & 0.00 \\
Gerber Yogurt Blends Peach & 4.72 & 3.77 & 53.77 & 0.00 & 6.60 & 31.13 \\
Gerber Whole Wheat Cereal (Baby) & 0.44 & 1.32 & 4.82 & 93.42 & 0.00 & 0.00 \\
Gerber Oatmeal Single Grain & 0.00 & 0.00 & 22.22 & 77.78 & 0.00 & 0.00 \\
Gerber Rice Singe Grain & 0.00 & 42.03 & 2.90 & 55.07 & 0.00 & 0.00 \\
Earth's Best Organic Whole Grain Oatmeal & 0.00 & 1.25 & 5.63 & 93.13 & 0.00 & 0.00 \\
Gerber Oatmeal Single Grain Cereal & 0.00 & 0.00 & 56.25 & 43.75 & 0.00 & 0.00 \\
(Baby) & & & & & \\
\hline
\end{tabular}

Values for each sugar represent percentage of total sugar in product; * products listing HFCS as an ingredient.

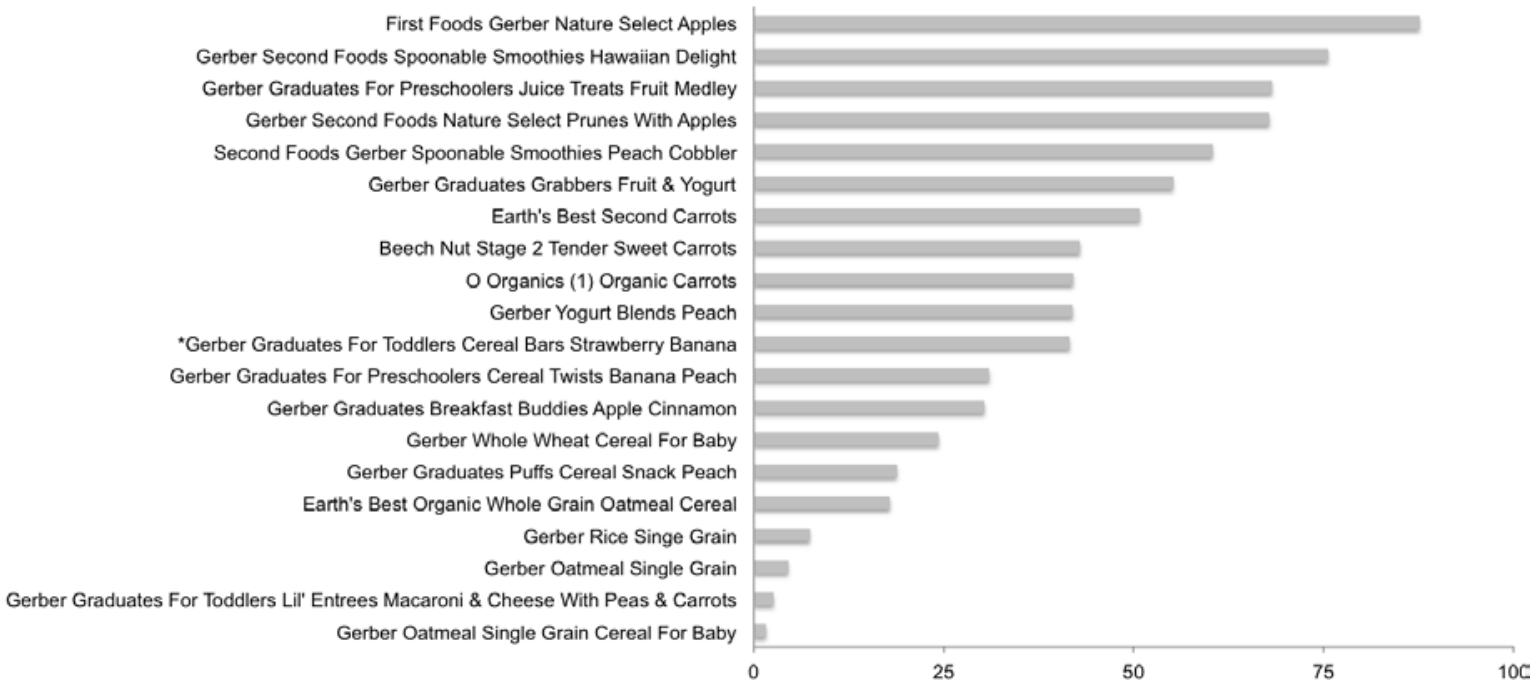

Figure 2. Percent of total calories from sugar: Baby Food. Values were derived from the following equation: $\%$ Total Calories from sugar $=\left[\left(\right.\right.$ Total $_{\text {Sugar }}$ Actual $\left._{\text {per serving }} \times 4\right) /$ Total Calories per serving] $\times 100$.

\subsubsection{Yogurt}

Mean percent of total calories per serving from sugars in the 20 yogurt samples tested was $51.9 \pm$ $13.9 \%$. Fourteen products contained $>50 \%$ of their total calories from sugar (Figure 3). Many yogurt 
products were sweetened with multiple sugars (sucrose, fructose and/or HFCS) and percentages of total sugars are listed in Table 1. All yogurts (excepting Chobani Raaspbery) contained added sugars with mean percent of total sugar from added sugar sources being $63.3 \pm 17.8 \%$ (Table S1). Yoplait Original Strawberry had a total sugars per serving of $27.2 \mathrm{~g} / \mathrm{s}$ and the mean sugar content per serving for all products was $14.4 \pm 5.6 \mathrm{~g} / \mathrm{s}$ (Table S2). The yogurt products analyzed ranged from 0 to $46 \%$ free fructose (Table 1). Nine of the 20 products tested reported fructose, of which one also listed HFCS, as an ingredient.

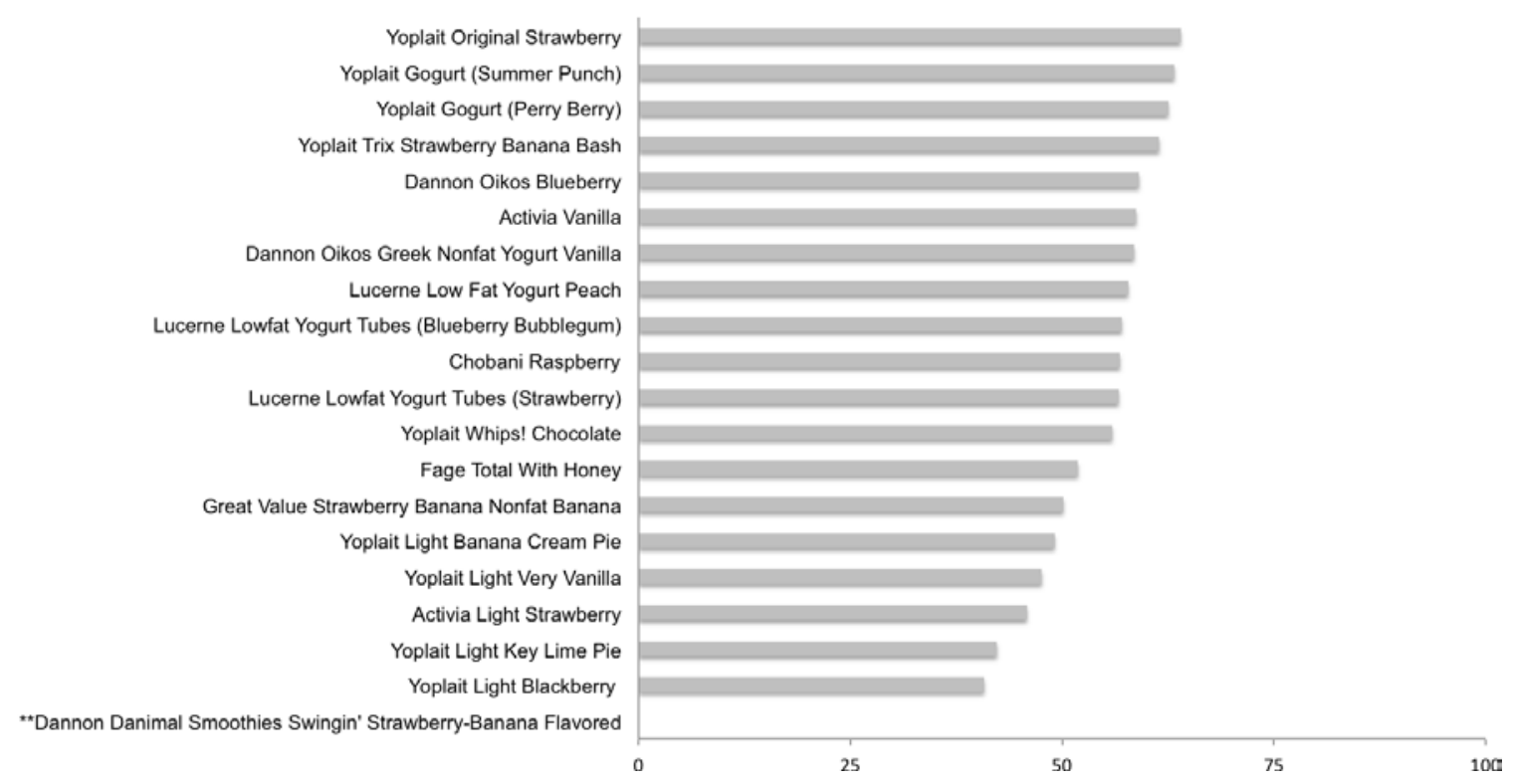

Figure 3. Percent of total calories from sugar: Yogurt. ** Label specifies no sugar. Values were derived from the following equation: \% Total Calories from sugar $=\left[\left(\right.\right.$ Total $_{\text {Sugar }}$ Actual per serving $\times 4$ )/Total Calories per serving] $\times 100$.

\subsubsection{Breakfast Cereals}

Mean percent of total calories per serving from sugars was $24.9 \pm 13.7 \%$ and twelve products contained $>20 \%$ of their total calories from sugar (Figure S1). The majority of products were mainly sweetened with sucrose (mean \% sucrose was $85.9 \pm 10.5 \%$ ), however two products (Cinnamon Toast Crunch and Corn Flakes) that did not list HFCS as an ingredient contained free fructose in excess of $15 \%$ of total sugars (Table 1). All cereal products (excepting Kashi Go Lean Crunch) contained added sugars with mean percent of total sugar from added sugar sources being $90.5 \pm 8.4 \%$ (Table S2). Most cereal products contained less than $10 \mathrm{~g}$ of total sugars per serving, however Kellogg's Frosted Flakes contained $23.8 \mathrm{~g} / \mathrm{s}$ of total sugar (Table S3). Only one cereal product listed HFCS as an ingredient (Essential Everyday Crispy Rice).

\subsubsection{Pre-Packaged Baked Goods}

Mean percent of total calories per serving from sugar was $34.9 \pm 7.6 \%$ with all products containing $>20 \%$ of their total calories from sugars (Figure S2). Baked goods were primarily sweetened with sucrose (mean \% of total sugars from sucrose was $67.1 \pm 24.9 \%$ (Table 1)). All baked goods products contained added sugars with mean percent of total sugar from added sugar sources being $93.4 \pm 11.6 \%$ 
(Table S2). Mean total sugars per serving in baked goods was relatively high at $18.9 \pm 11.8 \mathrm{~g} / \mathrm{s}$ and four products (Hostess Twinkies, Hostess Donettes Crunch, Hostess Ho Hos and Hostess Sno Balls) had total sugars values of $\geqslant 30 \mathrm{~g} / \mathrm{s}$ (Table S3). Low-calorie optioned baked goods (Keebler Right Bites and Fiber One brownies) tended to have higher fructose than sucrose levels.

\subsection{Comparison of Gas Chromatography Measured Total Sugar with Nutrient Label Data}

Total sugars per serving was calculated for all products, using the equation listed in Table S1, for comparison with per serving values listed on the individual product nutrient labels. This comparison could not be made in a subset of products due to no sugar per serving values being present on the label (formulas), specific gravity not being measured (one yogurt product) or the label specifying zero sugar (one baby food product). The percent difference from labeled sugar varied widely within and between grocery product groups (Table 2). As mentioned, formulas did not provide sugar per serving values on nutrition labels preventing the comparison in this group. Baby foods ranged from $88 \%$ less to $82 \%$ more total sugar than listed and similar observations were made for yogurts ( $24 \%$ less to $11 \%$ more), breakfast cereals ( $21 \%$ less to $18 \%$ more) and packaged baked goods (15\% less to $28 \%$ more).

Table 2. Comparison of per serving sugar: Laboratory measured vs. nutrition label value.

\begin{tabular}{cc}
\hline Infant Formula & \% Difference From Label \\
\hline * NA & $*$ NA \\
\hline Baby Foods & \% Difference From Label \\
\hline Gerber Whole Wheat Cereal For Baby & 82.40 \\
O Organics (1) Organic Carrots & 31.35 \\
Gerber Graduates Puffs Cereal Snack Peach & 17.60 \\
Gerber Rice Singe Grain & 10.40 \\
First Foods Gerber Nature Select Apples & 9.54 \\
Earth's Best Second Carrots & 3.95 \\
Gerber Second Foods Spoonable Smoothies Hawaiian Delight & 1.70 \\
Gerber Graduates For Preschoolers Juice Treats Fruit Medley & 0.31 \\
Gerber Graduates Breakfast Buddies Apple Cinnamon & -3.42 \\
Gerber Yogurt Blends Peach & -4.60 \\
Second Foods Gerber Spoonable Smoothies Peach Cobbler & -7.09 \\
Gerber Graduates Grabbers Fruit \& Yogurt & -8.00 \\
Gerber Second Foods Nature Select Prunes With Apples & -8.62 \\
Gerber Graduates For Toddlers Cereal Bars Strawberry Banana & -9.04 \\
Gerber Graduates For Preschoolers Cereal Twists Banana Peach & -11.43 \\
Beech Nut Stage 2 Tender Sweet Carrots & -14.12 \\
Gerber Oatmeal Single Grain & -66.25 \\
** Earth's Best Organic Whole Grain Oatmeal Cereal & -70.00 \\
Gerber Oatmeal Single Grain Cereal For Baby & -88.00 \\
Gerber Graduates For Toddlers Lil' Entrees Macaroni \& Cheese With Peas \& & - \\
\hline$\quad$ Carrots & \\
\hline & \\
\hline & \\
\hline
\end{tabular}


Table 2. Cont.

\begin{tabular}{|c|c|}
\hline Yogurt & \% Difference From Label \\
\hline Yoplait Gogurt (Summer Punch) & 10.72 \\
\hline Yoplait Light Banana Cream Pie & 10.50 \\
\hline Yoplait Trix Strawberry Banana Bash & 9.77 \\
\hline Yoplait Gogurt (Perry Berry) & 9.44 \\
\hline Yoplait Original Strawberry & 8.80 \\
\hline Yoplait Light Very Vanilla & 7.10 \\
\hline Chobani Raspberry & 4.68 \\
\hline Yoplait Whips! Chocolate & 1.70 \\
\hline Dannon Oikos Blueberry & 1.05 \\
\hline Activia Light Strawberry & 0.29 \\
\hline Lucerne Lowfat Yogurt Tubes (Blueberry Bubblegum) & -0.16 \\
\hline Lucerne Lowfat Yogurt Tubes (Strawberry) & -0.80 \\
\hline Dannon Oikos Greek Nonfat Yogurt Vanilla & -2.50 \\
\hline Lucerne Low Fat Yogurt Peach & -3.67 \\
\hline Yoplait Light Key Lime Pie & -4.80 \\
\hline Activia Vanilla & -4.95 \\
\hline Yoplait Light Blackberry & -8.20 \\
\hline Great Value Strawberry Banana Nonfat Banana & -8.82 \\
\hline Fage Total With Honey & -23.97 \\
\hline *** Dannon Danimal Smoothies Swingin' Strawberry-Banana Flavored & - \\
\hline Breakfast Cereal & \% Difference From Label \\
\hline Lucky Charms & 17.72 \\
\hline Life & 13.60 \\
\hline Kellogg's Frosted Flakes & 13.43 \\
\hline Cheerios & 12.00 \\
\hline Golden Grahams & 8.81 \\
\hline Frosted Cheerios & 7.33 \\
\hline Cocoa Puffs & 6.38 \\
\hline Kix & 1.00 \\
\hline Honey Nut Cheerios & 0.65 \\
\hline Cinnamon Toast Crunch & 0.42 \\
\hline Frosted Mini Wheats Little Bites & 0.00 \\
\hline Rice Chex & -0.10 \\
\hline Trix & -0.18 \\
\hline Kellogg's Froot Loops & -4.57 \\
\hline Honey Bunches Of Oats & -5.00 \\
\hline Kellogg's Special K & -6.22 \\
\hline Essential Everyday Crispy Rice & -6.50 \\
\hline Kashi Go Lean Crunch & -10.31 \\
\hline Safeway Kitchens Crispy Rice & -13.38 \\
\hline Corn Flakes & -21.60 \\
\hline
\end{tabular}


Table 2. Cont.

\begin{tabular}{cc}
\hline Packaged Baked Goods & \% Difference From Label \\
\hline Entenmann's Classic Glazed Buttermilk Donuts & 28.25 \\
Hostess Donettes & 19.26 \\
Hostess Donettes Crunch & 11.63 \\
Entenmann's Little Bites & 10.59 \\
Little Debbie Little Muffins Blueberry & 8.69 \\
Bimbo Mini Mantecadas Mini Muffins & 7.89 \\
Entenmann's Little Bites & 4.88 \\
Mini Chips Ahoy Bite Size & 3.67 \\
Kellogg's Special K Pastry Crisps Chocolaty Delight & 2.14 \\
Nabisco Fig Newtons & 1.53 \\
Mini Oreo Bite Size & 0.97 \\
Hostess Ho Hos & -0.37 \\
Kellogg's Pop-Tarts Frosted Strawberry & -0.55 \\
Safeway Graham Crackers & -1.19 \\
Fiber One 90 Calorie Brownies & -1.88 \\
Little Debbie Cosmic Brownies With Chocolate Chip Candy & -2.61 \\
Hon Calorie Right Bites Keebler Mini Brownies & -4.00 \\
Hostess Sno Balls & -4.23 \\
\hline Habisco Nilla Wafers & -8.64 \\
\hline Hostess Twinkies & -15.74 \\
\hline
\end{tabular}

Unable to calculate due to: * no sugar per serving value on label; ** Label specifies zero sugar; *** specific gravity not measured to determine actual sugar content per serving.

\section{Discussion}

This is the first comprehensive study to use laboratory-based chemical analyses to determine the actual content and composition of sugars in infant formulas, baby foods and a broad spectrum of popular grocery items that children are often exposed to. A principal finding of this study is that $74 \%$ of the 100 selected samples analyzed contained $\geqslant 20 \%$ of total calories per serving from sugars. Second, of the products analyzed $83 \%$ contained at least one source of added sugar and amongst these products $74 \%$ of total sugar content came from added versus intrinsic sugar sources. Additionally, nutrient label data often grossly under or overestimated actual sugar content, an observation most prominent in baby foods, which range from $88 \%$ less to $82 \%$ more total sugar than listed. These findings build upon prior work $[11,12]$, which determined the sugar content of sugar sweetened beverages and juices with laboratory analyses, and further supports the concept that children may be exposed to greater than anticipated consumption of daily added sugars. Current evidence suggests that children should limit sugar intake to less than $10 \%$ of total energy $[18,19]$. Given our findings in the selected products analyzed, it could be difficult to achieve these recommended levels of sugar consumption. This could put children at risk of future health consequences.

Of the 20 infant formulas that were analyzed, nine contained $\geqslant 20 \%$ of total calories per serving from sugars (seven products had $\geqslant 93 \%$ of total sugar from lactose while two had $\sim 45 \%$ and $80 \%$ of total sugar from sucrose). Although these measures are not directly comparable to the sugar content (lactose) in 
mature human breastmilk, which although variable generally remains close to $7 \%[8,20]$, it is important to point out the differences in sugar composition. Most formulas list sugar in their ingredients, which classifies them as products with added sugar. The added $v s$. intrinsic sugar classification is difficult to interpret in formulas, thus it is perhaps more relevant to dichotomize these products by lactose $v s$. any other added sugar. As mentioned above, in products designed to mimic the composition of breastmilk the majority of this "added" sugar comes from lactose, the naturally occurring sugar in human milk. Although free fructose detected in formulas was low, in seven products sucrose was the dominant added sugar representing nearly $86 \%$ of total added sugar. This was most notable in formulas designed for children with lactose intolerance or other gastrointestinal sensitivities. Of note, only two of the 20 infant formula products listed values for total sugars in grams per serving on their nutrition labels, making interpretation of sugar content and consumption impossible. It is not well understood how exposure to sucrose, and therefore fructose, in infancy impacts development and health progression. It is, however, well established that early life exposure to sugars other than lactose can influence taste preferences, satiety and health longitudinally $[21,22]$ and numerous animal studies show that exposure to fructose in early-life, including maternal transmission during pregnancy and lactation, can have long-lasting obesity promoting effects in the offspring [23]. These findings become important when considering baby foods as well, given that the mean percent of total calories per serving from sugar was $38.6 \pm 25.5 \%$ and seven baby food products contained $>50 \%$ of their total calories from sugar. This is consistent with a recent nutrient label analysis showing that $45 \%$ of 240 packaged baby foods had sugar levels in excess of $20 \%$ of calories from sugar [24]. Given the abundance of data linking added sugar consumption to disease risk it is imperative that medical professionals and consumers understand the actual sugar content of these products so as to make informed decisions in using them to nourish children early in life.

The yogurt samples that we analyzed contained $51.9 \% \pm 13.9 \%$ of total calories per serving from sugars. Seven of the yogurt products that were analyzed were directly marketed towards children, five of which contained $>10 \mathrm{~g}$ of total sugars per serving (Yoplait Whips! Chocolate, Dannon Danimal Strawberry-Banana Flavored, Yoplait Gogurt Perry Berry, Yoplait Trix Strawberry Banana Bash and Yoplait Gogurt Summer Punch), which translates to over 50\% of total calories per serving from sugar. The variability in fructose content and overall composition was large in yogurt products. Interestingly, several "light" or "low fat" products had more fructose than sucrose, which would be consistent with the common use of HFCS-90 as an unrestricted ingredient to sweeten low calorie foods [25] or the use of additional free fructose to improve palatability in some low-calorie food products. Lactose is a naturally occurring disaccharide (glucose and galatose) in yogurt and, as such, is expected to contribute to total sugar content. However, the predominant sugar in the yogurt products analyzed was added sucrose. Notably, in six of the seven products marketed towards children $70 \%-80 \%$ of total sugar comes from added sucrose and fructose whereas 15\%-20\% came from lactose (see Table 1). Given such high added sugar content, specifically in yogurt products for children, some of the beneficial health effects of yogurt $[26,27]$ could be undermined by excessive sugar content.

Daily consumption of breakfast cereals is predictive of a lower body mass index [28-30]. However, the high sugar content (mean percent of total calories per serving from sugar was $24.9 \% \pm 13.7 \%$ ) we observe within some of these products may place children at a higher risk for poor metabolic outcomes. This is especially true given recent findings demonstrating that up to $5 \%$ of children often consume 
more than 10 servings per day of cereal [30]. Nineteen cereal products contained added sugar as $\sim 91 \%$ of total sugar, predominantly in the form of sucrose. Our analyses are consistent with another recent report by the Environmental Working Group [31] that noted very high total and added sugar content in similar cereal products. Cereals may be a viable source of vitamins and fiber, but when consumed in excess, exposure to added sucrose and fructose in some products could be deleterious to metabolic health. The Children's Food and Beverage Advertising Initiative has established maximum per-serving total sugar contents for fruit, vegetable and grain products [32]. Most cereal products exceed these recommendations that stipulate less than $10 \mathrm{~g} / \mathrm{s}$ should come from sugar.

This study does have some limitations that merit discussion. The selection of the products analyzed was not based on their actual contribution to the diet of children. Unfortunately there are no data for how little or how much each of these products contribute to the diets of infants and young children. The list of products analyzed is by no means comprehensive, however it is likely representative of the average sugar profiles amongst products in each category, which is supported by another study that analyzed products in a similar category [31]. Although our data do not provide direct evidence that these products contribute to total sugar consumption in children, we feel that they strongly support our suggestion that adherence to current dietary guidelines may be difficult given the abundance of total sugar in products that children commonly consume. Our selection of samples from grocery categories was not completely random. Rather, it was based on a strategy to systematically select products with HFCS as a listed or unlisted ingredient. This could potentially bias the analysis towards higher fructose concentrations, however given the pervasive use of HFCS and sucrose as added sugars in the industry it may be a realistic representation of the actual sugar composition of products in these categories. More analyses of products will be needed to determine this. Additionally, we were unable to make added $v s$. intrinsic sugar comparisons in some products with naturally occurring sugars (fruit and dairy) and HFCS. Our use of GC methodology to analyze sugars has been previously criticized $[33,34]$ for theoretically overestimating fructose concentrations resultant from not accurately capturing DP2+ sugars (an industry classification referring to the sum of maltose, maltotriose, and maltotetraose). Although the focus of this study is not fructose-centric, per se, it is worth noting that our prior work $[11,35]$ has replicated sugar values (specifically fructose) across a variety of methodologies, including high performance liquid chromatography (HPLC). The White et al. papers posit that the HPLC method employed in their work is superior. However, the HPLC method used is an internal, industry-utilized protocol adapted from methodologies utilized by the Corn Industries Research Foundation, Corn Refiners Association and the Sweetener Technical Committee [34], and developed by the International Society of Beverage Technologists (ISBT) [36] to measure sugar composition in a beverage matrix. This is a widely used method, however it is not readily apparent that the ISBT methods have been subject to validation and critical review outside of the confines of internal food and beverage-related industrial technical publications. GC is also extensively used in industry for similar sugar analysis purposes and although we cannot directly compare against HPLC-generated values for the samples in this study, we are confident that GC accurately captures the sugars that are the focus of this work, namely sucrose, fructose, glucose, galactose, maltose and lactose. We acknowledge that the field would greatly benefit from an independent body conducting a double-blinded assessment of the sugar composition of food and beverage items in order to resolve the ongoing discrepancy in the sugar composition of popular beverages and other 
products that children may be exposed to, which is of paramount importance to our understanding of nutritional factors associated with the major burden of obesity and diabetes in our population.

Our study has demonstrated that total sugars, in many cases, constitute well over $20 \%$ of total calories across a variety of products (including infant formula) that children regularly consume in early life. High levels of sugar consumption, specifically fructose, have been shown to be metabolically deleterious in humans as soon as 10 weeks [37-40]. Although some products were low in measurable free fructose (not sweetened with HFCS), sucrose does contribute substantially to fructose exposure once it is enzymatically degraded. Therefore, exposure in early life to food products high in added sugars, such as sucrose or fructose, could "add up" across food categories and contribute to elevated risk for serious metabolic health outcomes [40,41]. The American Heart Association recommends less than 12 $\mathrm{g} /$ day added sugars for persons consuming $1600 \mathrm{kcal} /$ day (representative of children $4-8$ years) and the Unites States Dietary Guidelines Advisory Committee stipulates that added sugars should constitute less than $10 \%$ of total calories per day $[42,43]$. Thus the chronic, long-term consumption of foods and beverages high in sugar above these guidelines represents a significantly risk to child health. Of note was the large variability in actual sugar per serving content versus the labeled per serving sugar values. Nearly one quarter (22\%) of all products included in the comparison had actual total sugar values that were either greater than $10 \%$ or less than $10 \%$ of labeled total sugar (Table 2). The massive variability detected in the actual versus labeled total sugar content of baby foods ( \pm over $80 \%$ difference from label) is especially alarming. These wide ranges illustrate the enormous variability present in products, which could be due to batch differences or errors when assessing nutrient composition for reporting to the FDA [17]. Many of the baby foods indicating no added sugar in their ingredients, had high sugar content. This is possibly due to the presence of other sugar sources (brown rice syrup, evaporated cane juice, mono- and diglycerides, invert sugar and fruit or fruit juice concentrate) that are not generally highlighted as sources of added sugar. Thus, in many products listing no added sugar there are still high levels of fructose and sucrose regardless of whether the source of the sugar is added or intrinsic, which is similar to recent findings in fruit juices [11]. This collective work further supports prior concern that nutrition databases, and thus the nutrition labels that they are based upon, may not accurately reflect true sugar content. This could potentially lead to the over or underestimations of sugar intake in research and clinical contexts. More importantly, these discrepancies are apparent in some foods developed for and marketed directly towards infants and toddlers. Given these findings, informing the consumer of the sugar content of foods children are exposed to, by appropriate labeling of total sugar content in addition to actual sugar composition, could lead to judicious use of sweetened foods [44] and assist in preventing future adverse health outcomes resultant from the overconsumption of sugar early in life.

\section{Conclusions}

In conclusion, this study provides new information on the sugar composition and overall content of a multitude of commonly consumed food products. Many products that are frequently marketed to and consumed by infants and young children contain sugars that are far in excess of what is considered nutritionally beneficial and/or different from that stated on nutrition label. Given the importance of early infant nutrition in developmental programming [6,23] and exposure to excess sugar in infancy being associated with adverse metabolic consequences in adolescence and adulthood, it is essential to limit 
added sugar consumption in infancy and for consumers to be aware of the sugar content in products for children. These findings provide further support for adding more comprehensive sugar labeling to food and beverage products, specifically those marketed to, or commonly consumed by, children.

\section{Acknowledgments}

We would like to thank Kelly Dumke for her assistance with sample selection, Covance for their support during the analysis and members of the Childhood Obesity Research Center at the University of Southern California for their assistance. This study was supported by: (a) The National Center for Research Resources and the National Center for Advancing Translational Sciences, National Institute of Health (NIH), through Grant Award Number TL1RR031992, (b) The Ruth L. Kirschstein National Research Service Award NIH grant number 2T32ES013678-06 and (c) The Robert C. and Veronica Atkins Foundation.

\section{Author Contributions}

R.W.W. and M.I.G.: Conception and design of the study; generation, collection, assembly, analysis and/or interpretation of data; and drafting or revision of the manuscript; approval of the final version of the manuscript.

\section{Conflicts of Interest}

The authors declare no conflicts of interest. The founding sponsors had no role in the design of the study; in the collection, analyses, or interpretation of data; in the writing of the manuscript, and in the decision to publish the results.

\section{References}

1. Marriott, B.P.; Olsho, L.; Hadden, L.; Connor, P. Intake of added sugars and selected nutrients in the United States, National Health and Nutrition Examination Survey (NHANES) 2003-2006. Crit. Rev. Food Sci. Nutr. 2010, 50, 228-258. [CrossRef] [PubMed]

2. Pan, L.; Li, R.; Park, S.; Galuska, D.A.; Sherry, B.; Freedman, D.S. A longitudinal analysis of sugar-sweetened beverage intake in infancy and obesity at 6 years. Pediatrics 2014, 134 (Suppl. S1), S29-S35. [CrossRef] [PubMed]

3. Niinikoski, H.; Ruottinen, S. Is carbohydrate intake in the first years of life related to future risk of NCDs? Nutr. Metab. Cardiovasc. Dis. 2012, 22, 770-774. [CrossRef] [PubMed]

4. Davis, J.N.; Koleilat, M.; Shearrer, G.E.; Whaley, S.E. Association of infant feeding and dietary intake on obesity prevalence in low-income toddlers. Obesity 2014, 22, 1103-1111. [CrossRef] [PubMed]

5. Kohlboeck, G.; Sausenthaler, S.; Standl, M.; Koletzko, S.; Bauer, C.P.; von Berg, A.; Berdel, D.; Krämer, U.; Schaaf, B.; Lehmann, I.; et al. Food intake, diet quality and behavioral problems in children: Results from the GINI-plus/LISA-plus studies. Ann. Nutr. Metab. 2012, 60, 247-256. [PubMed] 
6. Koletzko, B.; Brands, B.; Poston, L.; Godfrey, K.; Demmelmair, H. Early nutrition programming of long-term health. Proc. Nutr. Soc. 2012, 71, 371-378. [CrossRef] [PubMed]

7. Park, S.; Pan, L.; Sherry, B.; Li, R. The association of sugar-sweetened beverage intake during infancy with sugar-sweetened beverage intake at 6 years of age. Pediatrics 2014, 134 (Suppl. S1), S56-S62. [CrossRef] [PubMed]

8. Cooper, A.R.; Barnett, D.; Gentles, E.; Cairns, L.; Simpson, J.H. Macronutrient content of donor human breast milk. Arch. Dis. Child. Fetal Neonatal Ed. 2013, 98, F539-F541. [CrossRef] [PubMed]

9. Fleddermann, M.; Demmelmair, H.; Koletzko, B. Energetic efficiency of infant formulae: A review. Ann. Nutr. Metab. 2014, 64, 276-283. [CrossRef] [PubMed]

10. Cogswell, M.E.; Gunn, J.P.; Yuan, K.; Park, S.; Merritt, R. Sodium and sugar in complementary infant and toddler foods sold in the United States. Pediatrics 2015, 135, 416-423. [CrossRef] [PubMed]

11. Walker, R.W.; Dumke, K.A.; Goran, M.I. Fructose content in popular beverages made with and without high-fructose corn syrup. Nutrition 2014, 30, 928-935. [CrossRef] [PubMed]

12. Ventura, E.E.; Davis, J.N.; Goran, M.I. Sugar content of popular sweetened beverages based on objective laboratory analysis: Focus on fructose content. Obesity 2011, 19, 868-874. [CrossRef] [PubMed]

13. Hastings, G.; McDermott, L.; Angus, K.; Stead, M.; Thomson, S. The Extent, Nature and Effects of Food Promotion to Children: A Review of the Evidence; Technical Paper/Prepared for the World Health Organization: Geneva, Switzerland, 2007; Available online: http://apps.who.int/iris/handle/10665/43627-sthash.49PESWdI.dpuf (accessed on 12 June 2015).

14. Milburn, M.V.; Lawton, K.A. Application of metabolomics to diagnosis of insulin resistance. Annu. Rev. Med. 2013, 64, 291-305. [CrossRef] [PubMed]

15. Mason, B.; Slover, H. A Gas Chromatographic Method for the determination of Sugar in foods. $J$. Agric. Food Chem. 1971, 19, 551-554. [CrossRef]

16. Brobst, K.M. Gas Liquid chromatography of trimethylsilyl derivations. Methods Carbohydr. Chem. 1972, 6, 3-8.

17. Mary, M.; Bender, J.I.R.; Foster, D. McClure, Guidance for Industry: Nutrition Labeling Manual-A Guide for Developing and Using Data Bases; U.S. Food and Drug Administration: Silver Spring, MD, USA, 1998. Available online: http://www.fda.gov/Food/GuidanceRegulation/GuidanceDocumentsRegulatoryInformation/ LabelingNutrition/ucm063113.htm - stat_5 (accessed on 24 March 2015).

18. Te Morenga, L.; Mallard, S.; Mann, J. Dietary sugars and body weight: Systematic review and meta-analyses of randomised controlled trials and cohort studies. BMJ 2013, 346, e7492. [CrossRef] [PubMed]

19. WHO. Diet, Nutrition and the Prevention of Chronic Diseases; World Health Organization: Geneva, Switzerland, 2003.

20. Jenness, R. The composition of human milk. Semin. Perinatol. 1979, 3, 225-239. [PubMed]

21. Mennella, J.A. Ontogeny of taste preferences: Basic biology and implications for health. Am. J. Clin. Nutr. 2014, 99, 704s-711s. [CrossRef] [PubMed] 
22. Cornwell, T.B.; McAlister, A.R. Alternative thinking about starting points of obesity: Development of child taste preferences. Appetite 2011, 56, 428-439. [CrossRef] [PubMed]

23. Goran, M.I.; Dumke, K.; Bouret, S.G.; Kayser, B.; Walker, R.W.; Blumberg, B. The obesogenic effect of high fructose exposure during early development. Nat. Rev. Endocrinol. 2013, 9, 494-500. [CrossRef] [PubMed]

24. Elliott, C.D.; Conlon, M.J. Packaged baby and toddler foods: Questions of sugar and sodium. Pediatr. Obes. 2015, 10, 149-155. [CrossRef] [PubMed]

25. NAP. Food Chemical Codex, 4th ed.; National Academy Press: Washington, DC, USA, 1996; pp. 191-192.

26. Mozaffarian, D.; Hao, T.; Rimm, E.B.; Willett, W.C.; Hu, F.B. Changes in diet and lifestyle and long-term weight gain in women and men. N. Engl. J. Med. 2011, 364, 2392-2404. [CrossRef] [PubMed]

27. Wang, H.; Livingston, K.A.; Fox, C.S.; Meigs, J.B.; Jacques, P.F. Yogurt consumption is associated with better diet quality and metabolic profile in American men and women. Nutr. Res. 2013, 33, 18-26. [CrossRef] [PubMed]

28. Barton, B.A.; Eldridge, A.L.; Thompson, D.; Affenito, S.G.; Striegel-Moore, R.H.; Franko, D.L.; Albertson, A.M.; Crockett, S.J. The relationship of breakfast and cereal consumption to nutrient intake and body mass index: The National Heart, Lung, and Blood Institute Growth and Health Study. J. Am. Diet. Assoc. 2005, 105, 1383-1389. [CrossRef] [PubMed]

29. Bazzano, L.A.; Song, Y.; Bubes, V.; Good, C.K.; Manson, J.E.; Liu, S. Dietary intake of whole and refined grain breakfast cereals and weight gain in men. Obes. Res. 2005, 13, 1952-1960. [CrossRef] [PubMed]

30. Albertson, A.M.; Anderson, G.H.; Crockett, S.J.; Goebel, M.T. Ready-to-eat cereal consumption: Its relationship with BMI and nutrient intake of children aged 4 to 12 years. J. Am. Diet. Assoc. 2003, 103, 1613-1619. [CrossRef] [PubMed]

31. Environmental Working Group. Children's Cereals: Sugar by the Pound. Available online: http://www.ewg.org (accessed on 12 February 2015).

32. Children's Food and Beverage Advertising Initiative Council of Better Business Bureaus. Category-Specific Uniform Nutrition Criteria Documents. 2011. Available online: http://www.bbb.org/ (accessed on 5 April 2013).

33. White, J.S.; Hobbs, L.J.; Fernandez, S.Re. "Fructose content in popular beverages made with and without high fructose corn syrup". Nutrition 2015, 31, 417-418. [CrossRef] [PubMed]

34. White, J.S.; Hobbs, L.J.; Fernandez, S. Fructose content and composition of commercial HFCS-sweetened carbonated beverages. Int. J. Obes. 2015, 39, 176-182. [CrossRef] [PubMed]

35. Goran, M.I.; Ventura, E.E.; Davis, J. Response to the "Letter to the Editor by LJ Hobbs". Obesity 2011, 19, 687-688. [CrossRef]

36. International Society of Beverage Technologists. ISBT method 3.2-Saccharides in soft drinks. In Quality Guidelines and Analytical Procedures of High Fructose Syrup 42 and 55; International Society of Beverage Technologists: Dallas, TX, USA, 2013. 
37. Livesey, G.; Taylor, R. Fructose consumption and consequences for glycation, plasma triacylglycerol, and body weight: Meta-analyses and meta-regression models of intervention studies. Am. J. Clin. Nutr. 2008, 88, 1419-1437. [PubMed]

38. Teff, K.L.; Grudziak, J.; Townsend, R.R.; Dunn, T.N.; Grant, R.W.; Adams, S.H.; Keim, N.L.; Cummings, B.P.; Stanhope, K.L.; Havel, P.J. Endocrine and metabolic effects of consuming fructose- and glucose-sweetened beverages with meals in obese men and women: Influence of insulin resistance on plasma triglyceride responses. J. Clin. Endocrinol. Metab. 2009, 94, 1562-1569. [CrossRef] [PubMed]

39. Hudgins, L.C.; Parker, T.S.; Levine, D.M.; Hellerstein, M.K. A dual sugar challenge test for lipogenic sensitivity to dietary fructose. J. Clin. Endocrinol. Metab. 2011, 96, 861-868. [CrossRef] [PubMed]

40. Wang, D.D.; Sievenpiper, J.L.; de Souza, R.J.; Chiavaroli, L.; Ha, V.; Cozma, A.I.; Mirrahimi, A.; Yu, M.E.; Carleton, A.J.; di Buono, M.; et al. The effects of fructose intake on serum uric acid vary among controlled dietary trials. J. Nutr. 2012, 142, 916-923. [CrossRef] [PubMed]

41. David Wang, D.; Sievenpiper, J.L.; de Souza, R.J.; Cozma, A.I.; Chiavaroli, L.; Ha, V.; Mirrahimi, A.; Carleton, A.J.; di Buono, M.; Jenkins, A.L.; et al. Effect of fructose on postprandial triglycerides: A systematic review and meta-analysis of controlled feeding trials. Atherosclerosis 2014, 232, 125-133. [CrossRef] [PubMed]

42. Johnson, R.K.; Appel, L.J.; Brands, M.; Howard, B.V.; Lefevre, M.; Lustig, R.H.; Sacks, F.; Steffen, L.M.; Wylie-Rosett, J.; American Heart Association Nutrition Committee of the Council on Nutrition, Physical Activity, and Metabolism and the Council on Epidemiology and Prevention. Dietary sugars intake and cardiovascular health: A scientific statement from the American Heart Association. Circulation 2009, 120, 1011-1020. [PubMed]

43. Scientific Report of the 2015 Dietary Guidelines Advisory Committee. In Advisory Report to the Secretary of Health and Human Services and the Secretary of Agriculture; United States Department of Agriculture: Washington, DC, USA, 2015.

44. Food and Drug Administration. Code of Federal Regulations Title 21. Direct Food substances Affirmed as Generally Recognized as Safe. Database of Select Committee on GRAS Substances (SCOGS) Reviews; Report No. 50. FDA: Silver Spring, MD, USA, 1979.

(C) 2015 by the authors; licensee MDPI, Basel, Switzerland. This article is an open access article distributed under the terms and conditions of the Creative Commons Attribution license (http://creativecommons.org/licenses/by/4.0/). 